\title{
Effect of Terminal Bond on Configurational Statistics of Poly(dimethylsiloxane)
}

\author{
Linxi ZHANG \\ Department of Physics, Hangzhou University, \\ Hangzhou, 310028, People's Republic of China
}

(Received January 25, 1994)

\begin{abstract}
A modified FCM (Flory, Crescenzi, and Mark) model is developed to study the configurational statistics of poly(dimethylsiloxane) (PDMS) chain. In the model, the theoretical molar cyclization equilibrium constants $K_{x}$ for small dimethylsiloxane ring are in agreement with the experimental data, and the theoretical characteristic ratio $C_{n}=\left\langle R^{2}\right\rangle / n l^{2}$ and its temperature coefficient for long ring are close to the experimental results.

KEY WORDS Configurational Statistics / Poly(dimethylsiloxane) Chain /

Rotational Isomeric State Model /
\end{abstract}

Silicone polymers are used in a variety of commercial applications inoluding lubricants, defoamers, greases, resins, and elastomers. ${ }^{1}$ Poly(dimethylsiloxane) (PDMS) is one of the most important silicone elastomers. PDMS has some unique configurational properties due to differing bond angles $\mathrm{Si}-\mathrm{O}-\mathrm{Si}$ and $\mathrm{O}-$ $\mathrm{Si}-\mathrm{O}$. The resulting configurational statistics of PDMS chains have been widely studied by many investigators. The most widely used approach for calculations of average statistical properties is based upon the rotational isomeric state (RIS) theory. ${ }^{2-8}$ The rotational isomeric state model of PDMS was set up by Flory, Crescenzi, and Mark (FCM), and their model satisfactorily reproduces the experimentally observed unperturbed mean-square end-to-end distance $\left\langle R^{2}\right\rangle$ and its temperature coefficient $\mathrm{d} \ln \left\langle R^{2}\right\rangle / \mathrm{d} T$. The FCM model also gives a reasonable account of the experimentally measured mean-square dipole moments $\left\langle\mu^{2}\right\rangle$. The molar cyclization equilibrium constants $K_{x}$ of PDMS were first calculated by Flory and Semlyen. ${ }^{9}$ However, a serious disagreement is present between experimentally observed ${ }^{10}$ cyclization probabilities for oligomers of
PDMS and predictions of the FCM model. The cyclization equlibrium constants $K_{x}$ measured for the oligomers with $x=4-6$ repeat unit turn out to be about 4 orders of magnitude higher than those computed based on the complete enumeration of the cyclic condormations of the FCM model. Recently, Bahar et al. ${ }^{8}$ developed a new rotational isomeric state approach to study the configurational statistics of PDMS. This model yields satisfactory agreement with experiments on mean-square unperturbed end-to-end distance, mean-square dipole moment and molar cyclization equilibrium constants $K_{x}(x=4-11)$ for dimethylsiloxane oligomers. But it cannot account for the positive temperature coefficient of the unperturbed chain dimensions. We notice that this model is only used to calculate the meansquare unperturbed end-to-end distance and its temperature coefficient for infinite chains because eq $12-15$ of ref 8 are obtained in the limit of $n \rightarrow \infty$. However, their approach yields a negative temperature coefficient of about $-2.34 \times 10^{-4} \mathrm{deg}^{-1}$ for the infinite chains. If the molar cyclization equilibrium constants $K_{x}$ with $x=4-11 \quad(n=2 x)$ are 
calculated using the statistical weight factors $\sigma, \omega, \omega^{\prime}, \tau$, and $\tau^{\prime}$ defined by eq $12-15$ of ref 8 , this may be a wrong, because eq $12-15$ are obtained from eq $6-11$ in the limit of $n \rightarrow \infty$. In this paper, a modified FCM model is developed to investigated the configurational statistics of PDMS.

\section{MODEL}

In the modified FCM rotational isomeric state model of PDMS, each skeletal bond is assigned to either a trans $(\phi=0)$, gauche ${ }^{+}$ $\left(\phi=120^{\circ}\right)$ or gauche $^{-}\left(\phi=240^{\circ}\right)$ state and mutual interdependence of adjacent pairs of bond rotational states is taken into account by means of two statistical weight matrices $U_{i \mathrm{a}}$ and $U_{i \mathrm{~b}}$. The matrix $U_{i \mathrm{a}}$ applies to rotational about pairs of bonds centred on oxygen atoms and the matrix $U_{i \mathrm{~b}}$ applies to rotational states about pairs of bonds centred on silicon atoms, thus,

$$
\begin{array}{r}
U_{i \mathrm{a}}=\left[\begin{array}{ccc}
1 & \sigma_{i} & \sigma_{i} \\
1 & \sigma_{i} & \sigma_{i} \omega_{i} \\
1 & \sigma_{i} \omega_{i} & \sigma_{i}
\end{array}\right] \\
U_{i \mathrm{~b}}=\left[\begin{array}{ccc}
1 & \sigma_{i} & \sigma_{i} \\
1 & \sigma_{i} & \sigma_{i} \omega_{i}^{\prime} \\
1 & \sigma_{i} \omega_{i}^{\prime} & \sigma_{i}
\end{array}\right]
\end{array}
$$

The configuration partition function of PDMS is given by

$$
Z=\left(\begin{array}{lll}
1 & 0 & 0
\end{array}\right) \prod_{i=1}^{x-1}\left(U_{i \mathrm{a}} U_{i \mathrm{~b}}\right)\left[\begin{array}{l}
1 \\
1 \\
1
\end{array}\right]
$$

and the mean-square end-to-end distance (or the mean-square dipole moment) may be calculated by serial multiplication of generator supermatrices as ${ }^{10,11}$

$$
\left\langle f^{2}\right\rangle=2 Z^{-1} g^{*} \prod_{i=1}^{x-1}\left(\mathscr{G}_{i \mathrm{a}} \cdot \mathscr{G}_{i \mathrm{~b}}\right) g
$$

where $\vec{f}$ is the bond vector $\vec{l}$ (or the bond dipole moment $\vec{m}$ ). For PDMS chain,

$$
\begin{aligned}
& l=l_{0}\left[\begin{array}{l}
1 \\
0 \\
0
\end{array}\right], \quad l_{0}=1.63 \AA \\
& m=m_{0}\left[\begin{array}{l}
1 \\
0 \\
0
\end{array}\right], \quad \begin{aligned}
m_{0}(\mathrm{Si}-\mathrm{O}) & =-m_{0}(\mathrm{O}-\mathrm{Si}) \\
& =0.60 D=\mu_{0}
\end{aligned}
\end{aligned}
$$

and

$$
\theta_{\mathrm{a}}=\angle \mathrm{OSiO}=70^{\circ}, \quad \theta_{\mathrm{b}}=\angle \mathrm{SiOSi}=37^{\circ}
$$

The Jacobson and Stockmayer theory ${ }^{12,13}$ provides the following expression for the molar cyclization equilibrium constants $K_{x}$ (in mol $1^{-3}$ ) for cyclics produced as eq 5

$$
K_{x}=\bar{W}_{\mathrm{x}} / 2 N_{\mathrm{A}} x
$$

where $\bar{W}_{x}$ represents the density of end-to-end vector $\vec{r}$ in the region $\vec{r} \cong 0$ (in mol $\mathrm{dm}^{-3}$ ) for an $x$-meric chain, and $N_{\mathrm{A}}$ is the Avogadro constant. $\bar{W}_{x}$ is given by

$$
\bar{W}_{x}=\left((4 / 3) \pi r^{3}\right)^{-1} \cdot Z_{r} / Z
$$

$Z_{r}$ is the sum of statistical weights for all chain conformations with terminal atoms separated by less than or as much as $r \AA$.

Molar cyclization equilibrium constants $K_{x}$ for small cyclic dimethylsiloxane $\left(\left(\mathrm{CH}_{3}\right)_{2} \mathrm{SiO}\right)_{x}$ with $x=4-6$ calculated using the FCM model are far lower than those found experimentally. Therefore, for a small dimethylsiloxane ring, it is necessary to take account of the mutual interdependence of bond rotational states further down the chain in the FCM model. The statistical weight parameters $\omega_{i}$ and $\omega_{i}^{\prime}$ in our model are different from the FCM model for the small dimethylsiloxane ring. For a long ring, they are the same as the FCM model. The difference between the FCM model and our model is that, in the FCM, statistical weight factors are the same for long and small rings, and in our model, are different.

\section{RESULTS AND DISCUSSION}

Molar cyclization equilibrium constants $K_{x}$ 
for small cyclic dimethylsiloxane $\left(\left(\mathrm{CH}_{3}\right)_{2} \mathrm{SiO}\right)_{x}$ $(x=4-6)$ are calculated by computing distances between the terminal silicon and oxygen atoms of the corresponding open chain molecules $\left(\left(\mathrm{CH}_{3}\right)_{2} \mathrm{SiO}\right)_{x}$ in all $3^{2 x-3}$ conformations, and using the FCM model. The results are that $\log K_{x}$ with $x=4-6$ and $r=3 \AA$ are $-4.04,-3.73$, and -3.42 , respectively. However, the experimental results are -0.744 , -1.02 , and -1.49 , respectively, which are about 4 orders of magnitude higher than those computed by the complete enumeration of the cyclic conformations of the FCM model. Small cyclic dimethylsiloxane $\left(\left(\mathrm{CH}_{3}\right)_{2} \mathrm{SiO}\right)_{4}(x=4)$ with the following sequences of rotational states $\mathrm{tg}^{+} \mathrm{g}^{-} \mathrm{g}^{+} \mathrm{g}^{-} \mathrm{g}^{+}$and $\mathrm{tg}{ }^{-} \mathrm{g}^{+} \mathrm{g}^{-} \mathrm{g}^{+} \mathrm{g}^{-}$are separated by less than $r=1.25 \AA$. If statistical weight factors $\omega_{i}$ and $\omega_{i}^{\prime}$ are increased, the theoretical molar cyclization equilibrium constants $K_{x}$ may be close to the experiment constants. For $x=6-8$, the theoretical molar cyclization equilibrium constants $K_{x}$ are in agreement with the experimental data, and the statistical weight factors $\omega_{i}$ and $\omega_{i}^{\prime}$ in our model are the same as in the FCM model. Table I lists the statistical weight factors deduced from the above considerations. Nonbonded interaction depends on distances of the atoms. Because of the differing bond angle $\mathrm{Si}-\mathrm{O}-\mathrm{Si}$ and $\mathrm{O}-\mathrm{Si}-\mathrm{O}$, the interaction energy of the group consisting of atoms may be different for long and small rings. This leads to different matrices $U_{i \mathrm{a}}$ and $U_{i \mathrm{~b}}$ for small $i$.

Figure 1 displays the characteristic ratio of the mean-square end-to-end distance $C_{n}=$

Table I. Energy parameters $\left(\mathrm{kcal} \mathrm{mol}^{-1}\right)^{\mathrm{a}}$

\begin{tabular}{rll}
\hline \multicolumn{1}{c}{$i$} & $E_{\omega_{i}}$ & $E_{\omega_{i}^{\prime}}$ \\
\hline 1 & 0.0 & 0.0 \\
2 & 0.0 & 0.0 \\
3 & 0.1 & 0.1 \\
4 & 0.3 & 0.5 \\
5 & 0.6 & 1.0 \\
$\geqslant 6$ & 1.05 & $\infty$
\end{tabular}

${ }^{\mathrm{a}} E_{\sigma_{i}}=0.85\left(\mathrm{kcal} \mathrm{mol}^{-1}\right)$. $\left\langle R^{2}\right\rangle / n l^{2} v s . n(n=2 x)$. The curve is drown on the basis of the modified model for PDMS chains $\mathrm{CH}_{3}\left(\mathrm{Si}\left(\mathrm{CH}_{3}\right)_{2} \mathrm{O}\right)_{x} \mathrm{Si}\left(\mathrm{CH}_{3}\right)_{2}$ at $T=$ $343 \mathrm{~K}$. For small $n$, the characteristic ratio $C_{n}$ is less than that calculated by the FCM model. For large $n$, the difference is insignificant. For example, the characteristic ratio $C_{n}$ of $n=600$ is 6.43 , which is less than that using the FCM model by $0.2 \%$. We also calculated the temperature coefficient $\mathrm{d} \ln \left\langle R^{2}\right\rangle / \mathrm{d} T$. The experimental values range from $0.28 \times 10^{-3}$ $\operatorname{deg}^{-1}$ to $0.78 \times 10^{-3} \mathrm{deg}^{-1} \cdot 2,14,15$ Figure 2 shows a plot of $\ln \left\langle R^{2}\right\rangle$ vs. $T$ for temperature ranging from $323 \mathrm{~K}$ to $363 \mathrm{~K}$. The slope of

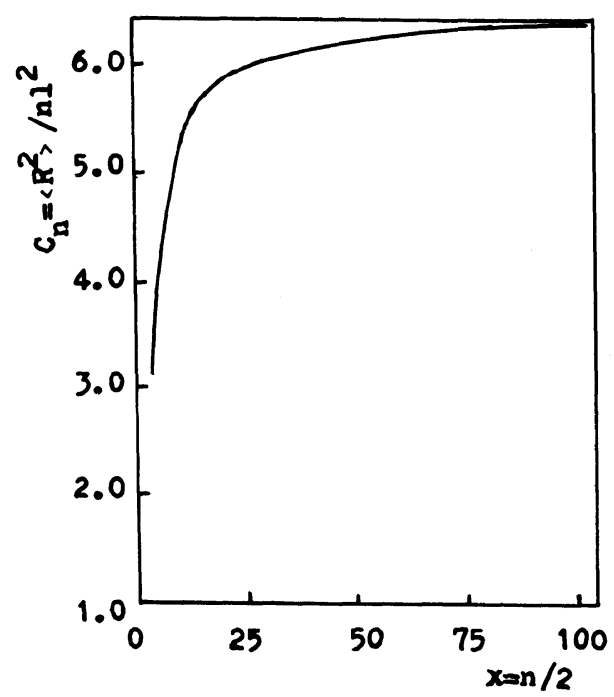

Figure 1. Dependence of $C_{n}=\left\langle R^{2}\right\rangle / n l^{2}$ on $x=n / 2$, at $T=343 \mathrm{~K}$.

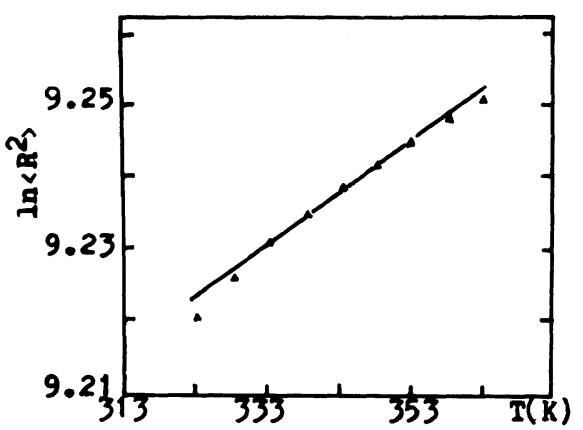

Figure 2. Plot of $\ln \left\langle R^{2}\right\rangle$ vs. temperature for a PDMS chain 600 bonds in length. 


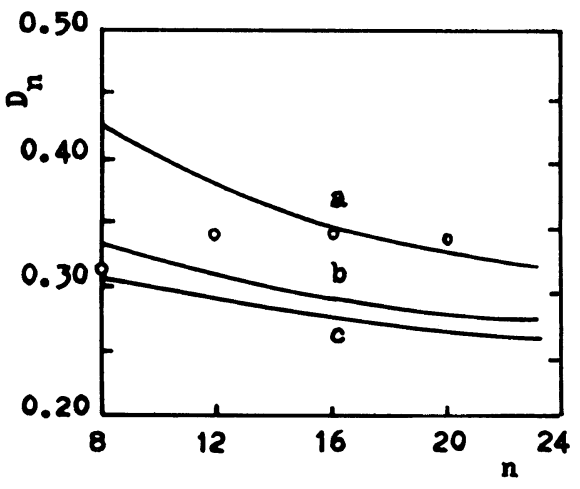

Figure 3. Dependence of $D_{n}\left(D_{n}=\left\langle\mu^{2}\right\rangle / n \mu_{0}^{2}\right)$ on $n$ at $T=303 \mathrm{~K}$. The dots are the experimental results from Sutton and Mark. ${ }^{16}$ a, Bahar et al.; b, this work; c, FCM model.

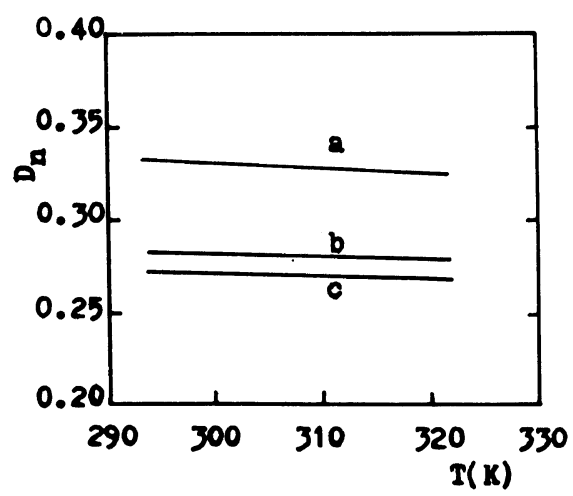

Figure 4. Plot of $D_{n} v s$. temperature for $n=20$. a, Bahar et al.; b, this work; c, FCM model.

this line is the temperature coefficient and we obtain $0.71 \times 10^{-3} \mathrm{deg}^{-1}$ for a chain of length 600 bonds. It should be emphasized that we calculated a positive temperature coefficient, a very necessary condition for this molecule, since all experiments showed a positive number the because a positive temperature coefficient explains so many of the elastic and flexible properties of PDMS.

The dependence of $D_{n}, D_{n}=\left\langle\mu^{2}\right\rangle / n \mu_{0}^{2}$, (where $\left\langle\mu^{2}\right\rangle$ is the mean-square dipole moment, and $\mu$ is the dipole of a $\mathrm{Si}-\mathrm{O}$ bond,) on the number $n$ of skeletal bonds is shown in Figure 3. The dots represent experimental measurements by Sutton and Mark. ${ }^{16}$ The line

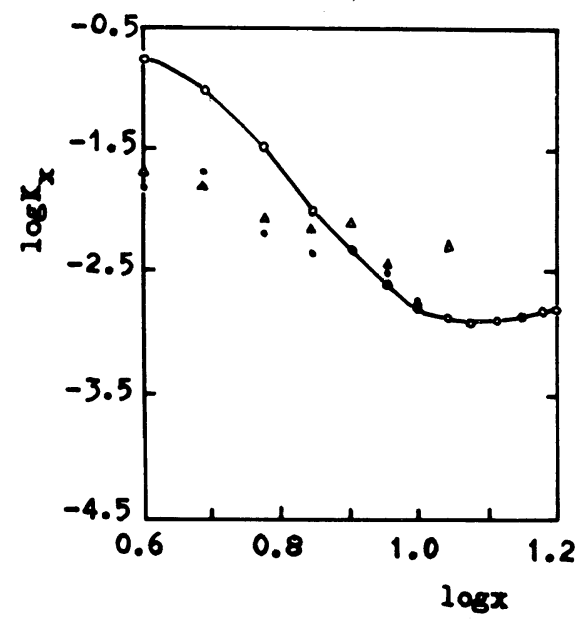

Figure 5. Theoretical molar cyclization equilibrium constants $K_{x}$ for cyclic dimethylsiloxane at $T=383 \mathrm{~K}$ (O) calculated by the direct computational method. The corresponding experimental values ${ }^{11}$ are denoted as $(O)$, and the results from Bahar et al. are denoted as $(\triangle)^{8}$

b was theoretically obtained using our model. The present theoretical yields a larger than that using the FCM model, and less than that of Bahar et $a .^{8}$ In Figure 4, temperature dependence of $D_{n}$ for $n=20$ is also given. Our values are greater than those using the FCM model, and less that the theoretical results reported by Bahar et al. ${ }^{8}$

Figure 5 displays the results of the molar cyclization equilibrium constants $K_{x}$ from experiments (empty circles) by Brown and Slusarczuk $^{11}$ in an equilibrate in toluene solution at $383 \mathrm{~K}$. The dashed circles follow from our model $(x=4-10)$, in agreement with experiments, and the results are calculated for $r=1.25 \AA$.

The differing bond angles $\mathrm{O}-\mathrm{Si}-\mathrm{O}$ and $\mathrm{Si}-\mathrm{O}-\mathrm{Si}$ may lead to difference in conformation between a long dimethylsiloxane ring and a small one. In the rotational isomeric state (RIS) model, statistical weight factors $\sigma$ and $\omega$ are often obtained by comparing theoretical plots of $C_{n}$ against $\sigma$ (or $\omega$ ) with experimental data. ${ }^{10,17}$ In this paper, the energy parameters are determined by comparing theoretical $K_{x}$ against $\omega$ and $\omega^{\prime}$ with experimental data for 
small $x$. Although the statistical weight matrices are obtained approximately, the configurational statistics of PDMS in our model agree with the experimental data.

Acknowledgement. This work was supported by National Natural Foundation of China.

\section{REFERENCES}

1. F. W. Billmeyer, Jr., "Textbook of Polymer Science," Wiley-Interscience, New York, N.Y., 1984.

2. J. E. Mark and P. J. Flory, J. Am. Chem. Soc., 86, 138 (1964).

3. P. J. Flory, V. Crescenzi, and J. E. Mark, J. Am. Chem. Soc., 86, 146 (1964).

4. P. J. Flory and V. W. C. Chang, Macromolecules, 9, 33 (1976).

5. J. E. Mark, Macromolecules, 11, 627 (1987).

6. S. Bruckner and L. Malpezzi, Makromol. Chem., 183,
2033 (1982).

7. J. A. Darsey, Macromolecules, 23, 5274 (1990).

8. I. Bahar, I. Zuniga, R. Dodge, and W. L. Mattice, Macromolecules, 24, 2993; 2986 (1991).

9. P. J. Flory and J. A. Semlyen, J. Am. Chem. Soc., 88, 3209 (1966).

10. P. J. Flory, "Statistical Mechanics of Chain Molecules," Wiley-Interscience, New York, N.Y., 1969.

11. J. F. Brown and C. M. Slusarczuk, J. Am. Chem. Soc., 87, 931 (1965).

12. H. Jacobson and W. H. Stockmayer, J. Chem. Phys., 18, 1600 (1950).

13. H. Jacobson, C. O. Beckmann, and W. H. Stockmayer, J. Chem. Phys., 18, 1607 (1950).

14. A. Ciferri, Trans. Faraday Soc., 57, 846; 853 (1961).

15. J. E. Mark and E. Erman, "Rubberlike Elasticity A Molecular Primer," John Wiley \& Sons, New York, N.Y., 1988.

16. C. Sutton and J. E. Mark, J. Chem. Phys., 54, 5011 (1971).

17. A. Abe and J. E. Mark, J. Am. Chem. Soc., 98, 6468 (1976). 